\title{
024 IMPACT OF LOW DOSE RATE BRACHYTHERAPY IN CLINICALLY LOCALIZED PROSTATE CANCER: CARDINAL SANTOS MEDICAL CENTER EXPERIENCE
}

Michael Jeff B Fontano, Jason L Letran. Cardinal Santos Medical Center, Quezon City, Philippines

\subsection{6/bmjopen-2015-forum2015abstracts.24}

Background In patients diagnosed to have clinically localized prostate cancer, approximately $45 \%$ chose some form of radiation for their initial treatment, making radiation the most common initial treatment modality. Brachytherapy is one form of radiation treatment for prostate cancer with toxicity profile that is generally well tolerated by most patients.

Objectives The objective of the study is to determine the outcome in terms of overall survival (OS), disease specific survival (DSS) and biochemical relapse free survival (BRFS), and adverse events of low dose rate brachytherapy among Cardinal Santos Medical Center patients with clinically localized prostate cancer.

Methods A total of 188 patients with clinically localized prostate cancer treated with low dose rate brachytherapy were stratified based on risk factors and grouped as low risk $(n=62)$, intermediate risk $(n=71)$, and high risk $(n=55)$. Analysis of variance (ANOVA), chi-square test and Kaplan-Meier survival analysis were used to determine statistical significance. P-values less than 0.05 indicate significant difference. The statistical analyses were done using MS Excel 2007.

Result The OS of patients who underwent brachytherapy was 94.1\%. The BRFS was 95.2\%, 95.8\% and 89.1\%, respectively. The DSS was $100 \%, 97.2 \%$ and $98.2 \%$, respectively. The adverse events identified were generally well tolerated.

Conclusion The success rate of brachytherapy in clinically localized prostate cancer among Cardinal Santos Medical Center patients generally showed good outcomes in terms of overall survival, disease specific survival and biochemical relapse free survival. The difference in outcomes among the groups of patient may have been attributed to factors such as Gleason score, preoperative PSA levels and stage of prostate cancer at initial presentation. 